\title{
Estudio comparativo del comportamiento electroquímico del hierro en medio alcalino en presencia de lodos rojos y lodos grises. Efecto del $\mathrm{Al}^{3+}\left({ }^{\circ}\right)$
}

\author{
C.M. Abreu* , L. Freire*, X.R. Nóvoa*, G. Pena* y M.C. Pérez*
}

\begin{abstract}
Resumen
Los elevados volúmenes de residuos generados por los lodos rojos (LR), procedentes del proceso de obtención de la alúmina a partir de la bauxita y los lodos grises (LG), originados durante el proceso de la transformación del granito, suponen un grave problema medioambiental. Trabajos recientes de este grupo demuestran la eficacia de ambos tipos de lodos como inhibidores de la corrosión del hierro y acero en medio alcalino clorurado. Esta característica ha llevado al estudio individualizado de sus componentes mayoritarios y, dentro de éstos, el $\mathrm{Al}^{3+}$, elemento común presente en mayor proporción. El estudio mediante técnicas electroquímicas como la Voltametría Cíclica (VC) y la Espectroscopía de Impedancia Electroquímica (EIE), apoyadas en técnicas de análisis químico y superficial, ha permitido visualizar cómo el $\mathrm{Al}^{3+}$ es capaz de bloquear la superficie del acero en medio alcalino aumentando su resistencia al ataque por cloruros.
\end{abstract}

\section{Comparative study of the electrochemical behaviour of iron in alkaline media containing red mud and grey slurries. The effect of $\mathrm{Al}^{3+}$}

\begin{abstract}
Red mud (RM) is the main by-product of the Bayer process for alumina extraction from bauxite, and grey slurries (GS) are generated during the granite rocks transformation processes. The huge volume of those wastes generated every year suppose a serious environmental problem. Recent works by our group demonstrate the efficiency of both types of muds as corrosion inhibitors for iron and steel in alkaline media containing chlorides. The elemental analysis of these slurries shows that $\mathrm{Al}^{3+}$ is the main common element. Therefore, the aim of this paper is to study the effect of the soluble $\mathrm{Al}^{3+}$ in the electrochemical behaviour of iron in alkaline media, in presence and absence of chlorides. The electrochemical study using Cyclic Voltammetry (CV) and Electrochemical Impedance Spectroscopy (EIS) together with surface analysis techniques allowed to conclude that $\mathrm{Al}^{3+}$ blocks the surface of steel in alkaline media chloride increasing the steel resistance to chloride attack.
\end{abstract}

Keywords $\quad$ Red mud; Grey slurries; CV; EIS; Corrosion inhibitor.

\section{INTRODUCCIÓN}

Los lodos rojos (LR) y los lodos grises (LG) son los principales residuos resultantes de dos procesos industriales ampliamente extendidos: mientras los primeros, LR, se originan en la extracción de la alúmina a partir de la bauxita, los segundos, LG, se generan durante los procesos de transformación del granito (corte y pulido de la piedra) en los que se produce un intenso desgaste de la roca, convirtiéndose en polvo alrededor de un $30 \%$ de la misma. La figura 1 muestra el aspecto que presentan ambos tipos de lodos.
Debido a las grandes cantidades de ambos lodos que se generan $(470.000$ y $30.000 \mathrm{~T} /$ año, respectivamente, para LR y LG, sólo en Galicia) y su fuerte alcalinidad ( $\mathrm{pH} \approx 12.0$ ), representan un importante problema medioambiental en las áreas donde están implantadas las industrias y en las zonas donde se acumulan $^{[1}$ y 2$]$

Ese problema medioambiental ha hecho que se hayan dedicado una importante cantidad de esfuerzos al estudio de aplicaciones para poner en valor estos residuos y darles así una salida ${ }^{[3-5]}$. En trabajos recientes propios $^{[1,2,6 \text { y } 7]}$ se demuestra la eficacia de

\footnotetext{
(•) Trabajo recibido el día 20 de diciembre de 2006 y aceptado en su forma final el día 3 de noviembre de 2008.
}

* Universidade de Vigo, ETSEI, Campus Universitario, 36310 Vigo (España). 

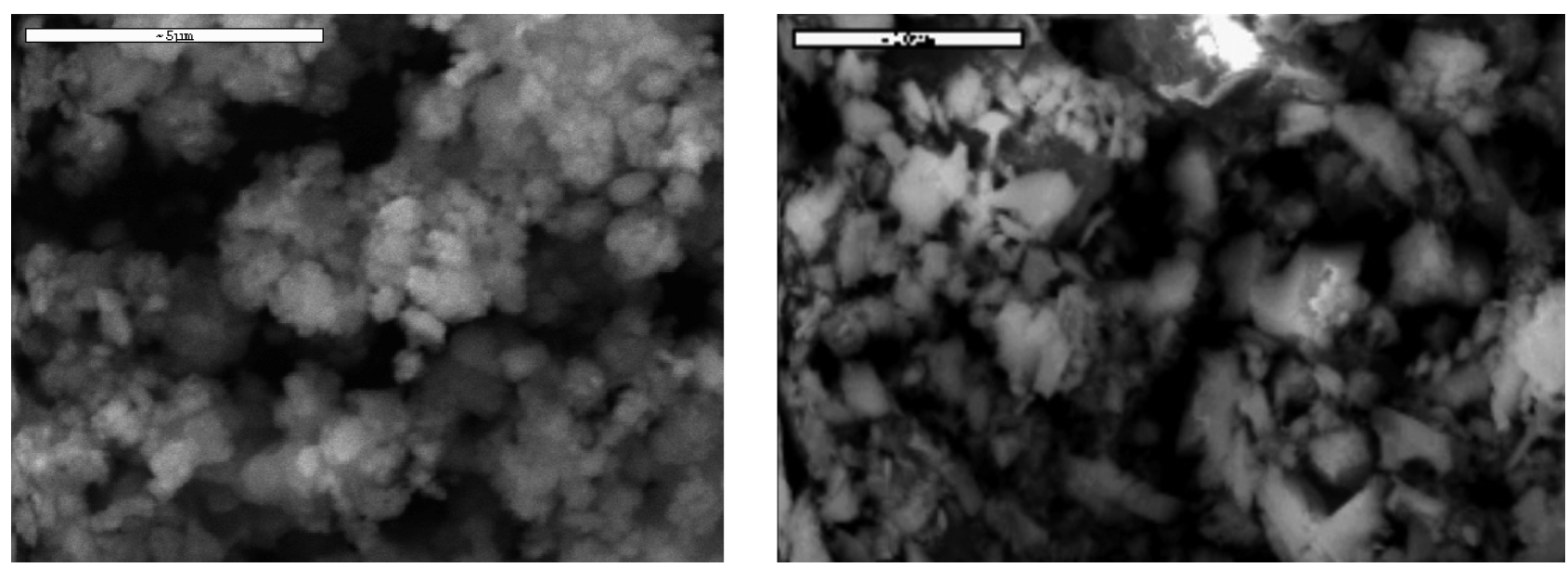

Figura 1. Imágenes de MEB de las partículas de LR (a) y LG (b).

Figure 1. SEM picture of RM (a) and GS (b) particles.

ambos tipos de lodos como inhibidores de la corrosión del hierro y acero en disoluciones alcalinas cloruradas. Sin embargo, se desconoce cuál es la sustancia o elemento activo responsable de esa actividad. El objetivo de este trabajo es analizar el efecto de uno de los elementos de composición mayoritario y común a ambos tipos de lodos: los iones aluminato.

\section{EXPERIMENTAL}

Para la caracterización química de los lodos se ha empleado un analizador elemental Fisons EA - 1108; para obtener resultados significativos, reproducibles y fiables es importante el empleo de muestras con un elevado grado de homogeneización, por lo que las muestras de lodos en bruto se homogeneizaron en un molino de bolas de tipo planetario.

Para los ensayos con lodos se ha utilizado una concentración de $2 \mathrm{~g} \cdot \mathrm{l}^{-1}$

A partir de ahí, este estudio comparativo se centró en el empleo de técnicas electroquímicas (Voltametría Cíclica y Espectroscopía de Impedancia Electroquímica) para analizar el papel que juega el $\mathrm{Al}^{3+}$ contenido en los LR y $\mathrm{LG}$ en el proceso de inhibición de la corrosión, utilizando una disolución alcalina de $\mathrm{NaOH}$ 0,1 M como electrólito de referencia, comparándola con la misma con diferentes adiciones de lodos y limaduras de Al metálico hasta su máximo de solubilidad (aproximadamente, 13,5 $\left.\mathrm{mg} \cdot \mathrm{l}^{-1}\right)$.

Además, se añadieron iones $\mathrm{Cl}^{-}$(en forma de $\mathrm{NaCl}$ ) para estudiar el efecto de los inhibidores frente a la presencia de estos iones despasivantes.

Las medidas electroquímicas se llevaron a cabo en una celda típica de tres electrodos:
- Electrodo de trabajo: las probetas se prepararon a partir de redondos de Fe de alta pureza (99,99\%, de Goodfellow), se desengrasaron con acetona y agua destilada y se recubrieron con una capa de pintura cataforética con el fin de delimitar el área de exposición, que fue de $0,2 \mathrm{~cm}^{2}$. Seguidamente, se embutieron en una resina epoxy bicomponente de curado en frío y se desbastaron en una lijadora-pulidora tipo Buëhler, empleando diferentes tamaños de grano en la lija: desde grado 500 a 1.200 .

- Electrodo de referencia: se eligió en función del medio. Uno de los electrodos más comúnmente empleados en la práctica habitual de las medidas electroquímicas es el de calomelanos saturado en $\mathrm{KCl}$ (SCE) con un potencial de $+0,244 \mathrm{~V}$ con respecto al del NHE Sin embargo, en el trabajo en disolución alcalina sin cloruros se utilizó como electrodo de referencia el $\mathrm{Hg} / \mathrm{HgO}$, puesto que su electrólito alcalino $(\mathrm{KOH} \mathrm{0,1} \mathrm{M})$ no contamina el medio de trabajo. Con el fin de facilitar la comparación de resultados se ha decidido referir en el texto todas las medidas al electrodo SCE.

- Electrodo auxiliar o contraelectrodo: se empleó una lámina de grafito de gran área (unos $\left.50 \mathrm{~cm}^{2}\right)$.

La celda electroquímica se introdujo en una caja de Faraday, para eliminar el ruido procedente de fuentes externas. Además, todos los ensayos electroquímicos fueron realizados a temperatura ambiente y abiertos a las atmósfera, utilizando un Potenciostato AUTOLAB (PGSTAT30) de la marca Ecochemie, controlado por ordenador.

En la Voltametría cíclica (VC) se efectuaron barridos desde $-1,4 \mathrm{~V}$ (próximo a la reacción de desprendimiento 
de $\mathrm{H}_{2}$ ) hasta $0,6 \mathrm{~V}$ vs. SCE (próximo a la reacción de evolución del $\mathrm{O}_{2}$ ), a una velocidad de barrido de 1 $\mathrm{mVs}^{-1}$, lo suficientemente lenta para permitir que tenga lugar la relajación de todos los procesos redox que ocurren en las películas pasivantes ${ }^{[8]}$.

Los ensayos de Espectroscopia de Impedancia Electroquímica (EIE) se utilizan para obtener información adicional de los procesos de electrodo y se realizaron en el correspondiente Potencial Libre de Corrosión. El rango de frecuencias analizado fue desde $10 \mathrm{kHz}$ a $1 \mathrm{mHz}$.

El control de las medidas y el registro de los datos obtenidos se efectuaron mediante las aplicaciones FRA (para EIE) y GPS (para VC) pertenecientes al software del AUTOLAB. El tratamiento y análisis de los resultados obtenidos se realizó a través de distintos programas informáticos desarrollados en el laboratorio y la hoja de cálculo Origin 7.0.

\section{RESULTADOSY DISCUSIÓN}

La tabla I muestra que los LR están constituidos básicamente por óxidos de hierro y aluminio (compuestos típicos de la bauxita) y los LG son ricos en silicatos y aluminatos (propios de las rocas graníticas) y contienen también óxidos de hierro y calcio procedentes de la granalla de acero y la cal añadidas durante el proceso de corte como abrasivos.

En el análisis elemental, también, podemos observar que el aluminio es elemento común presente en mayor porcentaje. En el caso de los LR una cantidad no cuantificada del aluminio detectado está formando parte de un silicato, una especie insoluble denominada "sodalita bayer"[7].

El aluminio es soluble en medio ácido, como $\mathrm{Al}^{3+}$, mientras que en medio básico se disuelve como iones aluminato (bien en forma hidratada, $\mathrm{Al}(\mathrm{OH})_{4}^{-}$ o anhidra, $\left.\mathrm{Al}(\mathrm{OH})_{2}^{-}\right)$. Por lo tanto, la solubilidad del $\mathrm{Al}^{3+}$ es dependiente del $\mathrm{pH}$ y varía entre los límites establecidos por su forma más soluble $\mathrm{Al}(\mathrm{OH})_{3}$ y la más insoluble $\left(\mathrm{Al}_{2} \mathrm{O}_{3} \cdot 3 \mathrm{H}_{2} \mathrm{O}\right)$, teniendo lugar el mínimo de solubilidad a $\mathrm{pH}=5,0^{[9]}$.
La finalidad del trabajo es saber si el importante efecto inhibidor encontrado en estos lodos se debe a la presencia de aluminato (la forma soluble en medio básico).

\subsection{Voltametría cíclica}

\subsubsection{Disoluciones alcalinas}

El comportamiento del Fe en medio alcalino con LR, LG y $\mathrm{Al}^{3+}$ fue analizado con VC.

En la figura 2 se comparan los octavos ciclos (cuando ya los voltamogramas son estables) de las medidas realizadas con lodos (LR y LG) y la disolución de referencia. Se han seleccionado estos ciclos puesto que, en ellos, ya está definida la morfología de la película ${ }^{[1]}$, de forma que se puede observar con claridad las posibles diferencias existentes cuando el Fe se encuentra en presencia de los lodos y cuando no lo está.

La morfología de la curva de referencia, presentada en la figura 2, es la típica de un electrodo de hierro en este medio ${ }^{[1]}$.

Un análisis de los voltamogramas presentados en la figura 2 muestra que:

- La carga asociada al pico de formación de la magnetita (Ec. 1)

$$
\begin{gathered}
3 \mathrm{Fe}(\mathrm{OH})_{2}+2 \mathrm{OH}^{-} \longrightarrow \mathrm{Fe}_{3} \mathrm{O}_{4} \cdot 4 \mathrm{H}_{2} \mathrm{O}+2 \mathrm{e}^{-} ; \\
\mathrm{E} \approx-0.9 \text { V vs. SCE }
\end{gathered}
$$

es menor en presencia de lodos, aunque no se observan diferencias en cuanto a morfología y posición del pico. (Fig. 2 a)). Este efecto puede ser atribuido a un bloqueo de la superficie metálica por parte de ambos tipos de lodos.

- En la zona de pasividad (Fig. 2 b)) no hay variaciones significativas en la densidad de corriente pero sí varía sensiblemente el rango de potenciales abarcado, que es mayor en presencia de LG y ostensiblemente más anódico el potencial de desprendimiento de oxígeno en presencia de LR.

Tabla I. Composición química mayoritaria de los lodos (\% peso)

Table I. Chemical composition (weight \%) for mud's main compounds

\begin{tabular}{ccccccc}
\hline & $\mathrm{Fe}_{2} \mathrm{O}_{3}$ & $\mathrm{Al}_{2} \mathrm{O}_{3}$ & $\mathrm{SiO}_{2}$ & $\mathrm{Na}_{2} \mathrm{O}$ & $\mathrm{CaO}$ & $\mathrm{TiO}_{2}$ \\
\hline $\mathrm{LR}$ & 40,37 & 28 & 8,5 & 5,98 & 4,82 & 10,10 \\
$\mathrm{LG}$ & 7,12 & 13 & 65,84 & 3,14 & 5,54 & 0,33 \\
\hline
\end{tabular}



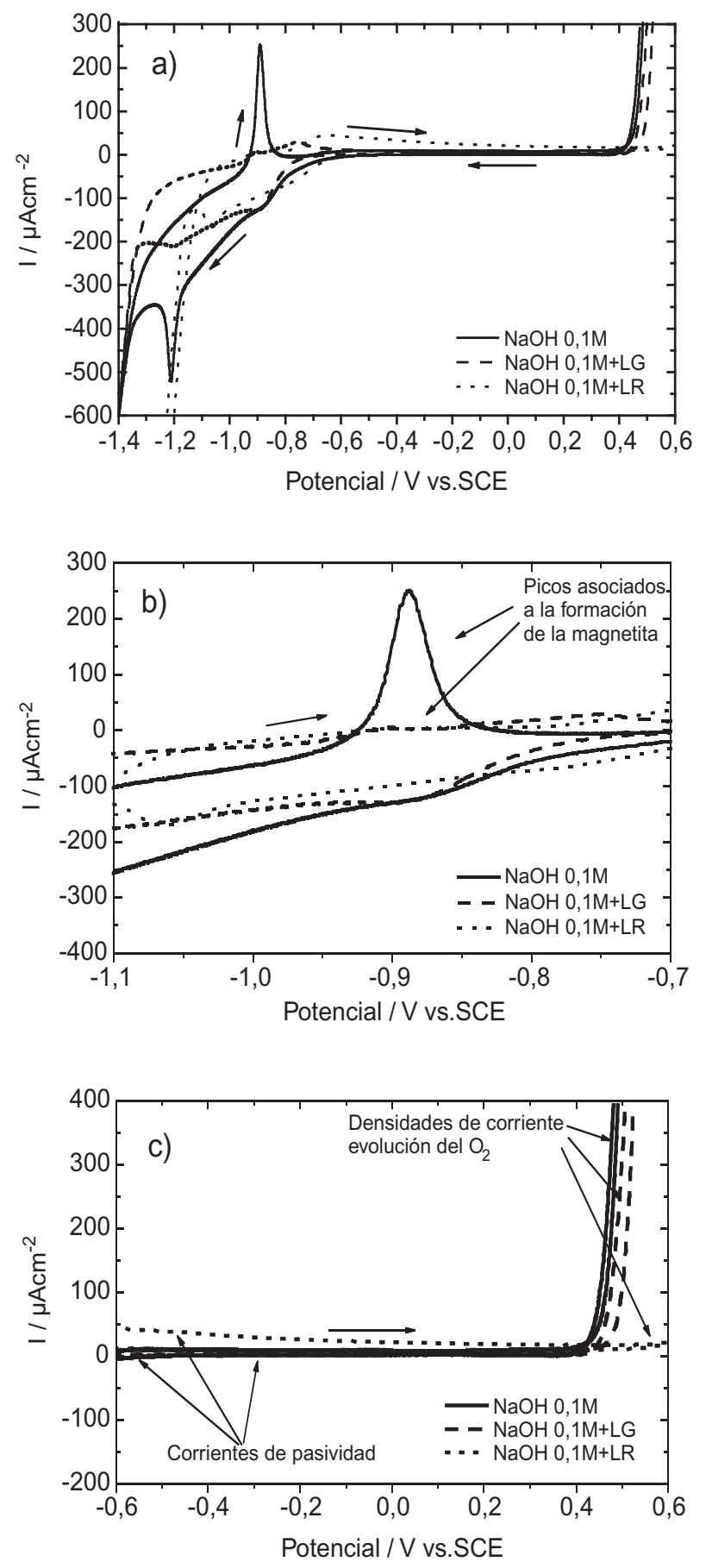

Figura 2. (a) Comparación de los voltamogramas correspondientes a los octavos ciclos obtenidos sobre el electrodo de $\mathrm{Fe}$ en una disolución de $\mathrm{NaOH} 0,1 \mathrm{M}$ con y sin lodos. $\mathrm{dE} / \mathrm{dt}=1$ $\mathrm{mV} . \mathrm{s}^{-1}$; (b) Detalle del pico asignado a la formación de la magnetita; (c) Detalle de las corrientes de evolución del $\mathrm{O}_{2}$.

Figure 2. (a) Comparison of the eighth cycles obtained for an iron electrode in $0.1 \mathrm{M} \mathrm{NaOH}$ with and without slurries. $d E / d t=1 \mathrm{mV} \mathrm{s}^{-1}$; (b) Detail of the magnetite formation peak; (c) Detail of the region corresponding to $\mathrm{O}_{2}$ evolution.
- En las curvas de vuelta se aprecia una importante diferencia en las corrientes de reducción (figura 2 a)). La corriente límite de $\mathrm{O}_{2}$, es mucho mayor en el caso de la disolución sin lodos.

Estos tres aspectos apuntan claramente a un bloqueo de la superficie del electrodo por parte de los dos tipos de lodos.

Si se representa el voltamograma de referencia junto al realizado en presencia de aluminatos (obtenida esta disolución por disolución del aluminio en medio alcalino), la situación es muy similar a la ya descrita, según se puede comprobar en la figura 3 .

La densidad de corriente del pico asignado a la formación de la magnetita y la densidad de corriente en el dominio catódico disminuyen considerablemente en presencia de los aluminatos (Figs. 3 a) y b)) y, además, el potencial de evolución del $\mathrm{O}_{2}$ se desplaza anódicamente (Fig. 3 c)), indicios, de nuevo, de un bloqueo de la superficie del electrodo de hierro.

El efecto que ejercen los lodos es, por lo tanto, análogo al que se observa con los aluminatos, por lo que la presencia del aluminio resulta esencial en la capacidad inhibidora de estos residuos.

\subsubsection{Disoluciones alcalinas con adición de cloruros}

La picadura es la forma de corrosión más dañina en los aceros y la presencia de cloruros en el medio es el factor principal para que se produzca. En el caso del acero en hormigón, la relación $\left[\mathrm{Cl}^{-}\right] /\left[\mathrm{OH}^{-}\right]$y el potencial son factores decisivos ${ }^{[10}$ y11] , por ello, se ha estudiado la capacidad de los aluminatos para inhibir la picadura. Intentando una aproximación a la situación de las armaduras de acero embebidas en hormigón cuando se encuentran sometidas a la acción agresiva del medio marino, se utilizó un medio de $\mathrm{pH}$ propio del hormigón sin carbonatar ( 13), con distintas relaciones de concentración de $\mathrm{NaOH}$ y $\mathrm{NaCl}$.

Se estudió, inicialmente, una relación $\left[\mathrm{Cl}^{-}\right] /\left[\mathrm{OH}^{-}\right]$ $=2$. En la disolución de referencia (sin adiciones) se produce la rotura local de la película, pasiva ya en el primer ciclo, situación que mejora considerablemente tanto con la adición de lodos como con la adición de aluminato, lo que demuestra que los aluminatos son capaces de evitar la corrosión localizada en el hierro. Este hecho se repite al aumentar la concentración de cloruros, tal y como ilustran las figuras 4 y 5 , donde se empleó una concentración superior de cloruros, una disolución más agresiva, $\left[\mathrm{Cl}^{-}\right] /\left[\mathrm{OH}^{-}\right]=5$, manteniendo siempre el $\mathrm{pH}$ alcalino. En este medio, el potencial de picadura del hierro es $\mathrm{E}_{\mathrm{p}}=0,16 \mathrm{~V}$, vs. SCE y el grado de histéresis en la curva de vuelta, sugieren la existencia de un gran número de picaduras 

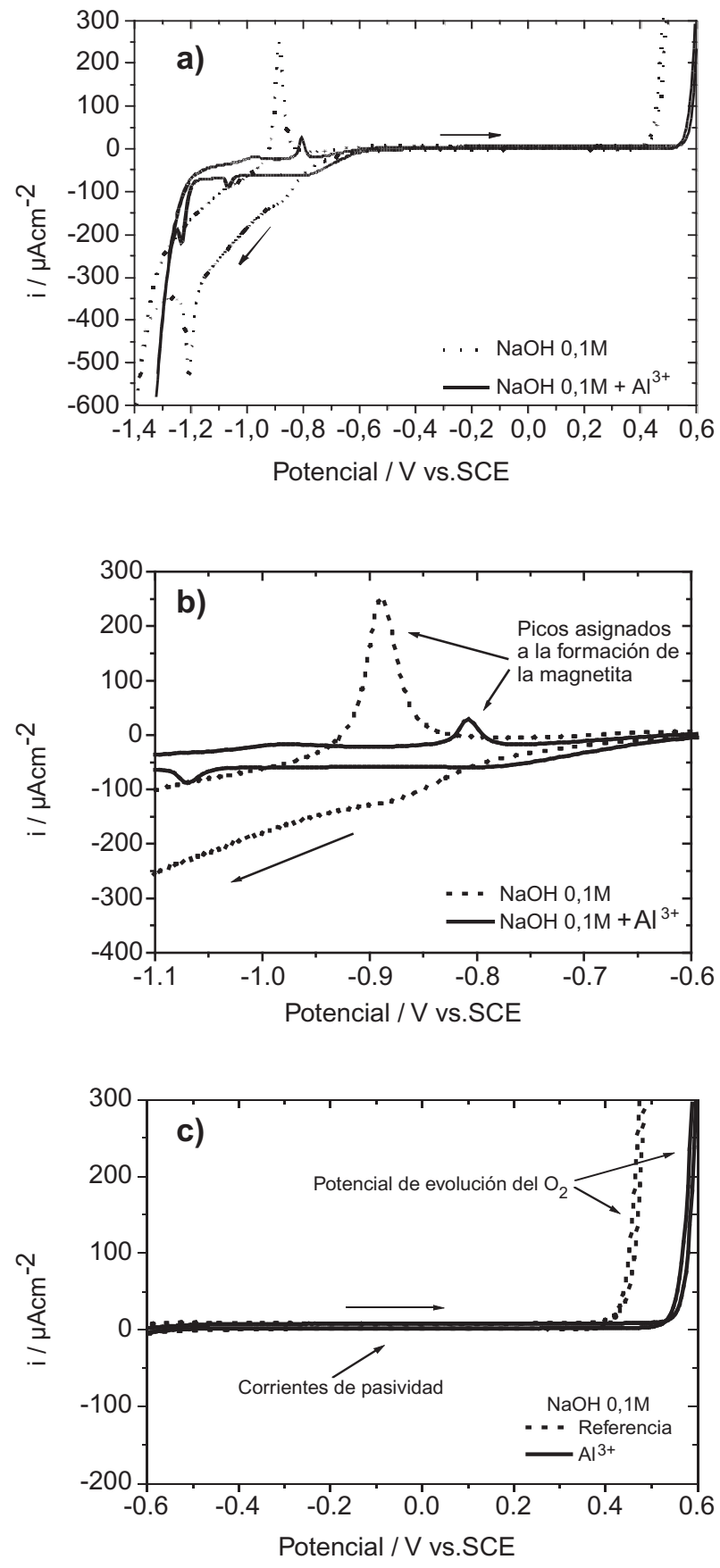

Figura 3. (a) Comparación de los voltamogramas correspondientes a los octavos ciclos obtenidos sobre el electrodo de Fe en una disolución de $\mathrm{NaOH} 0,1 \mathrm{M}$ con y sin adición de $\mathrm{Al}^{3+} 0,5$ $\mathrm{mM}$. $\mathrm{dE} / \mathrm{dt}=1 \mathrm{mV} \cdot \mathrm{s}^{-1}$; (b) Detalle de los picos asignados a la formación de la magnetita; (c) Detalle de las corrientes de evolución del $\mathrm{O}_{2}$.

Figure 3. (a) Comparison of the eight cycles obtained for an iron electrode in $0.1 \mathrm{M} \mathrm{NaOH}$ with and without the addition of $A^{3+} 0.5 \mathrm{mM} . d E / d t$ $=1 \mathrm{mV} \cdot \mathrm{s}^{-1}$;(b) Detail of the magnetite formation peak;(c) Detail of the region corresponding to $\mathrm{O}_{2}$ evolution.

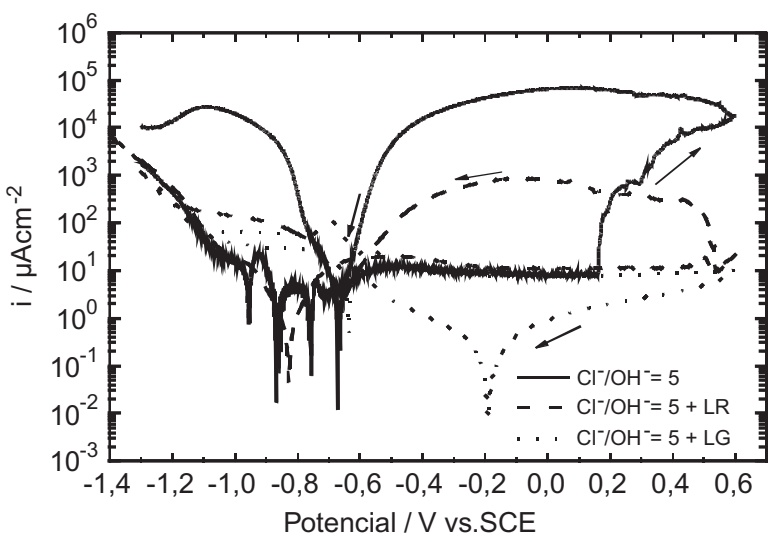

Figura 4. Comparación de los primeros ciclos obtenidos para un electrodo de Fe en una disolución de $\mathrm{NaOH} 0,1 \mathrm{M}+\mathrm{NaCl} 0,5 \mathrm{M}$ con y sin lodos. $\mathrm{dE} / \mathrm{dt}=1 \mathrm{mVs}^{-1}$.

Figure 4. Comparison of the first cycles obtained for iron electrode in $0.1 \mathrm{M} \mathrm{NaOH}+\mathrm{NaCl} 0.5 \mathrm{M}$ with and without slurries added. $d E / d t=1 \mathrm{mVs}^{-1}$.

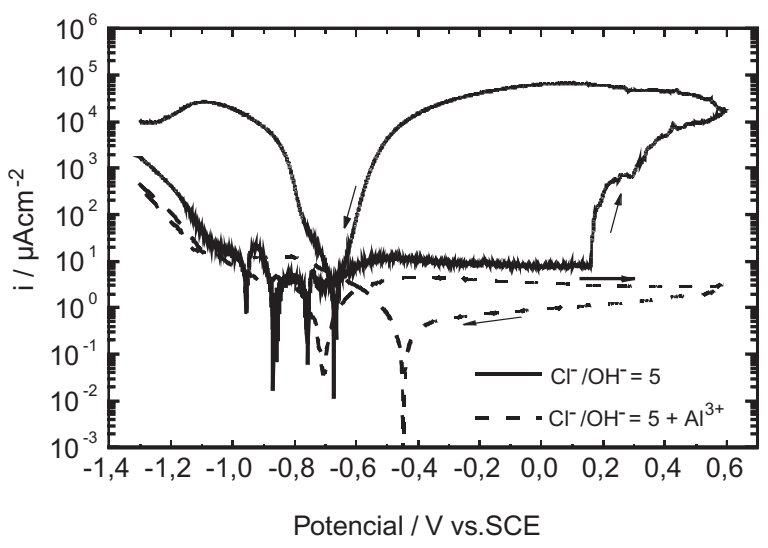

Figura 5. Comparación de los primeros ciclos obtenidos para un electrodo de Fe en una disolución de $\mathrm{NaOH} 0,1 \mathrm{M}+\mathrm{NaCl} 0,5 \mathrm{M}$ con y sin adición de $\mathrm{Al}^{3+} 0.5 \mathrm{mM}$. $\mathrm{dE} / \mathrm{dt}=1 \mathrm{mVs}^{-1}$.

Figure 5. Comparison of the first cycles obtained for iron electrode in $0.1 \mathrm{M} \mathrm{NaOH}+\mathrm{NaCl} 0.5 \mathrm{M}$ with and without $0.5 \mathrm{mM} \mathrm{A}^{3+}$. $d E / d t=1 \mathrm{mVs}^{-1}$.

formadas que se propagan rápidamente provocando una disolución catastrófica del metal. La densidad de corriente alcanzada $\left(15 \mathrm{~mA} \cdot \mathrm{cm}^{-2}\right)$ implica una alta velocidad de corrosión.

La figura 4 muestra que, en presencia de LR, la situación de corrosión evoluciona favorablemente. Se produce un proceso de corrosión por picadura pero a potenciales muy anódicos, por lo que el rango 
de pasividad es mayor. El $\mathrm{E}_{\mathrm{p}}$ se desplaza a potenciales positivos $\left(\mathrm{E}_{\mathrm{p}}=+0,55 \mathrm{~V}\right.$ vs. SCE) y aumenta la diferencia con respecto al potencial de repasivación $\left(\mathrm{E}_{\text {rep }}\right)$ lo que significa que el material, en este medio, presenta mejores características protectoras.

La carga asociada al proceso de corrosión inducida es considerablemente menor que en el caso de la disolución de referencia, la densidad de corriente alcanzada en la curva de vuelta es de $0,9 \mathrm{~mA} \cdot \mathrm{cm}^{-2}$, lo que implica una menor velocidad de disolución del metal.

La inspección visual de la superficie de las probetas de Fe tras el ensayo, muestra claramente la diferencia de ataque, en ambos casos. Mientras la muestra de Fe en la disolución de referencia presenta una disolución muy generalizada, con picaduras muy profundas que han avanzado por toda la superficie de la probeta, la muestra de Fe polarizada en el medio con LR, presenta una densidad menor de picaduras, de diámetro más pequeño y poco profundas, que implican una leve pérdida de material.

En las figuras 4 y 5 se puede observar, también, que tanto en presencia de LG (Fig. 4) como en presencia de aluminatos (Fig. 5) la situación de pasividad se mantiene incluso en el potencial más anódico explorado. Este estado permanece tras haber ciclado un total de ocho veces, lo que, de nuevo, demuestra el importante poder inhibidor que presentan los aluminatos.

\subsection{Potencial libre de corrosión}

La tabla II muestra que, para la disolución de referencia, al aumentar la concentración de cloruros (de 0,2 a 0,5 M) el potencial de corrosión disminuye. Este hecho puede entenderse, desde el punto de vista de la teoría del potencial mixto, como una activación del proceso anódico, acorde con el incremento en la concentración de agente despasivante. Sin embargo, en las disoluciones con aditivos (LR, LG y aluminato) los potenciales registrados son, globalmente, más catódicos a pesar del mejor comportamiento frente a la corrosión anteriormente comentado. La explicación hay que buscarla aquí, de nuevo, en la teoría del potencial mixto, pero del lado de la reacción catódica. Las figuras 2 a) y 3 a) muestran una importante disminución de la corriente de reducción de oxígeno en presencia de aditivos, por lo que el bloqueo de la reacción catódica debido a la presencia de aluminatos, LR y LG, justifica plenamente el desplazamiento observado.

El desplazamiento catódico del potencial de corrosión aumenta, según la literatura ${ }^{[10 \text { y } 11]}$. Sin embargo, habría que añadir que, según los datos aquí presentados, esta conclusión es válida siempre que el desplazamiento del potencial se deba a la inhibición de la reacción catódica. En este caso, la película pasiva, intacta químicamente, aumenta su grado de hidratación ${ }^{[12]}$, con lo que disminuye localmente la relación $\left[\mathrm{Cl}^{-}\right] /\left[\mathrm{OH}^{-}\right]$y, de ahí, la mayor resistencia a la formación de picaduras.

\subsection{Espectroscopía de impedancia electroquímica}

La EIE se utiliza para obtener información adicional de los procesos de electrodo y completar los datos obtenidos mediante VC.

La figura 6 se corresponde a los resultados de EIE en el potencial libre de corrosión para disoluciones alcalinas cloruradas $\left(\left[\mathrm{Cl}^{-}\right] /\left[\mathrm{OH}^{-}\right]=5\right)$ en presencia de lodos y de aluminatos. Estos datos experimentales fueron modelados utilizando un circuito equivalente, con dos constantes de tiempo (RC) distribuidas en cascada, según se muestra en la figura 7, y al que corresponde la impedancia de la ecuación 2.

$$
Z(\omega)=R_{e}+\frac{R_{1}}{\frac{1}{1+\left(Z_{2}(\omega) / R_{1}\right)}+\left(j \omega R_{1} C_{1}\right)^{\alpha_{1}}}
$$

\section{Tabla II. Evolución del potencial de corrosión $\left(\mathrm{V}_{\mathrm{vs} . \mathrm{SCE}}\right)$ para el electrodo de Fe en las diferentes disoluciones cloruradas estudiadas}
Table II. Evolution of the corrosion potential $\left(\mathrm{V}_{\mathrm{vs} . \mathrm{SCE}}\right)$ for the iron electrode in the chlorinated solutions studied

\begin{tabular}{|c|c|c|c|c|c|c|c|}
\hline \multicolumn{2}{|c|}{$\begin{array}{l}\mathrm{NaOH} 0,1 \mathrm{M} \\
+\mathrm{Cl}^{-}\end{array}$} & \multicolumn{2}{|c|}{$\begin{array}{c}\mathrm{NaOH} 0,1 \mathrm{M} \\
+\mathrm{Cl}^{-}+\mathrm{LR}\end{array}$} & \multicolumn{2}{|c|}{$\begin{array}{c}\mathrm{NaOH} 0,1 \mathrm{M} \\
+\mathrm{Cl}^{-}+\mathrm{LG}\end{array}$} & \multicolumn{2}{|c|}{$\begin{array}{c}\mathrm{NaOH} 0,1 \mathrm{M} \\
+\mathrm{Cl}^{-}+\mathrm{Al}^{3+}\end{array}$} \\
\hline $0,2 M$ & $0,5 M$ & $0,2 M$ & $0,5 M$ & $0,2 M$ & $0,5 M$ & $0,2 M$ & $0,5 M$ \\
\hline$-0,22$ & $-0,34$ & $-0,49$ & $-0,32$ & $-0,48$ & $-0,31$ & $-0,31$ & $-0,40$ \\
\hline
\end{tabular}




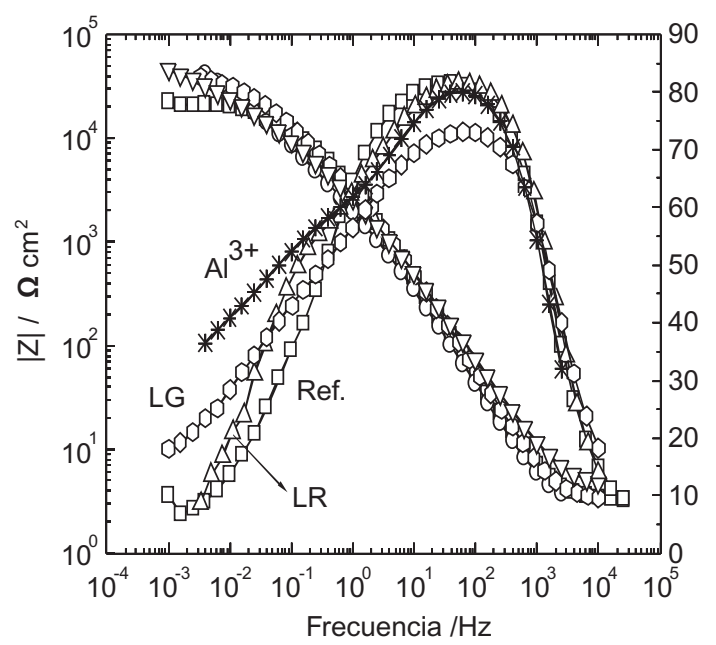

0
0
0
0
0
0
0
0
0
0
0
0
0
0
0
$\frac{1}{4}$
1

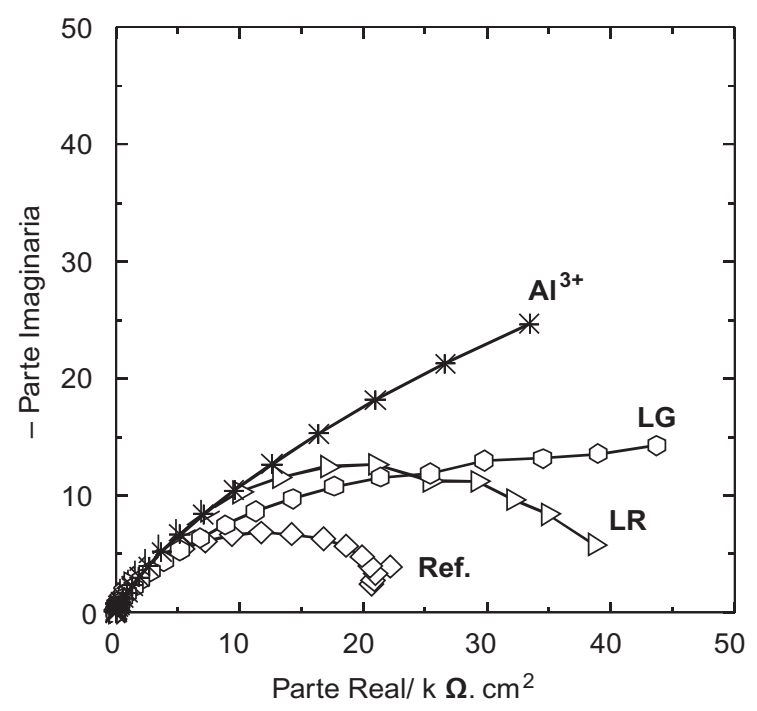

Figura 6. Diagramas de Bode (a) y Nyquist (b) de las medidas de impedancia obtenidas sobre un electrodo de $\mathrm{Fe}$ en $\mathrm{NaOH} 0,1 \mathrm{M}+\mathrm{NaCl} 0,5 \mathrm{M}$ con $\mathrm{y} \sin \mathrm{Al}^{3+} 0,5 \mathrm{mM}$.

Figure 6. Bode (a) and Nyquist (b) plots of the impedance measurements obtained for iron electrode in $\mathrm{NaOH} 0.1 \mathrm{M}+\mathrm{NaCl} 0.5 \mathrm{M}$ solution with and without $0.5 \mathrm{mM} \mathrm{A}{ }^{3+}$.

siendo

$$
Z_{2}(\omega)=\frac{R_{2}}{1+\left(j \omega R_{2} C_{2}\right)^{\alpha_{2}}}
$$

La constante de tiempo a alta frecuencia, $\mathrm{R}_{1} \mathrm{C}_{1}$, se asocia con la transferencia de carga a través de la interfase de capacidad de doble capa, $\mathrm{C}_{1}$, y la de baja frecuencia, $R_{2} C_{2}$, a los procesos redox que tienen lugar en la película pasivante. $\alpha_{1}$ y $\alpha_{2}$ son los grados de

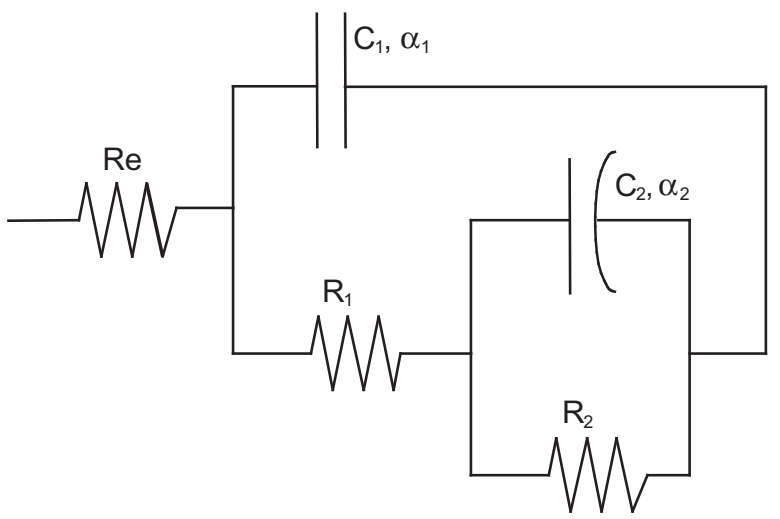

Figura 7. Circuito equivalente utilizado para el ajuste de los datos obtenidos en EIE. La impedancia global corresponde a la ecuación (2).

Figure 7. Equivalent circuit employed to model EIS data. The global impedance is given in equation (2).

dispersión de las constantes $\mathrm{R}_{1} \mathrm{C}_{1}$ y $\mathrm{R}_{2} \mathrm{C}_{2}$, respectivamente. Una descripción detallada del significado de estos parámetros se puede encontrar en la literatura ${ }^{[13]}$.

En los diagramas de Bode de la figura 6 a) destacan dos aspectos principales a saber:

- En el límite alta frecuencia no se aprecian diferencias entre las diferentes disoluciones, lo cual es indicativo de que el bloqueo de la superficie que ejercen LR, LG o aluminatos no comporta un factor resistivo de la película pasivante del tipo presente en ciertos aceros inoxidables $^{[14]}$, con lo que se confirma el mecanismo de control cinético sobre la reacción catódica argumentado anteriormente.

- Sí existe una importante diferencia en el ángulo de fase en el dominio de bajas frecuencias, dominio de frecuencias que está relacionado con la actividad redox en la película pasiva (espesor y grado de oxidación ${ }^{[8]}$ ).

En los diagramas de Nyquist representados en la figura $6 \mathrm{~b}$ ) se puede observar que el valor global de la impedancia es mayor en el caso de mayor contenido de $\mathrm{Al}^{3+}$ en disolución y decrece con la presencia de $L G$ y LR. Este hecho se refleja en el valor de $\mathrm{R}_{2}$ presentado en la tabla III, donde se comprueba que la resistencia aumenta al hacerlo la concentración de $\mathrm{Al}^{3+}$.

Según los datos de la tabla III, la capacidad asignada a la doble capa $\left(\mathrm{C}_{1}\right)$ es mayor en presencia de $\mathrm{Al}^{3+}$, lo que sugiere una acumulación de carga en la interfase, debido a una adsorción de los aluminatos. Sin embargo, no se ha observado ninguna incorporación de $\mathrm{Al}^{3+}$ a la película pasiva ni por Microscopía 
Tabla III. Parámetros de ajuste correspondiente a los gráficos de la figura 5 según la ecuación (1) (1)

Table III. Best fitting parameters for data given in figure 5, according to equation (1)

\begin{tabular}{cccccccc}
\hline $\mathrm{OH}^{-} / \mathrm{Cl}^{-}=\mathbf{5}$ & $\begin{array}{c}\mathbf{R}_{\mathrm{e}} \\
\Omega \cdot \mathbf{c m}^{2}\end{array}$ & $\begin{array}{c}\mathbf{R}_{1} \\
\mathbf{k} \Omega \cdot \mathbf{c m}^{2}\end{array}$ & $\begin{array}{c}\mathbf{C}_{1} \\
\boldsymbol{\mu} \mathbf{F} \cdot \mathbf{c m}^{-2}\end{array}$ & $\alpha_{1}$ & $\begin{array}{c}\mathbf{R}_{2} \\
\mathbf{k} \Omega \cdot \mathbf{c m}^{2}\end{array}$ & $\begin{array}{c}\mathbf{C}_{2} \\
\mathbf{m F} \cdot \mathbf{c m}^{-2}\end{array}$ & $\alpha_{2}$ \\
\hline Ref. & 3,2 & 7,6 & 43,1 & 0,9 & 16,6 & 1,9 & 0,7 \\
Ref. + LR & 3,2 & 4,4 & 35,0 & 0,9 & 36,6 & 1,3 & 0,7 \\
Ref. + LG & 5,1 & 16 & 27,0 & 0,9 & 50,0 & 1,4 & 0,5 \\
Ref. + Al ${ }^{3+}$ & 3,1 & 0,3 & 53,7 & 0,9 & 92,5 & 0,3 & 0,5 \\
\hline
\end{tabular}

Electrónica ni por XPS, lo que permite avanzar la formación de una película protectora de aluminatos, de carácter gelatinoso ${ }^{[15]}$, que desaparece una vez la muestra se retira de la disolución y se lava.

La disminución en $\mathrm{R}_{1}$ que se observa en la tabla III, a medida que aumenta la concentración de $\mathrm{Al}^{3+}$, es coherente con la variación de los potenciales de corrosión para estos sistemas reflejada en la tabla II, puesto que un menor potencial supone una mayor influencia del proceso redox $\mathrm{Fe}^{2+} / \mathrm{Fe}^{0}[14]$ y $\mathrm{Fe}^{0} / \mathrm{Fe}^{2+}$

La capacidad de baja frecuencia, $\mathrm{C}_{2}$, en el dominio de potenciales correspondiente a la pasividad, está relacionada con el proceso redox descrito en la ecuación (4) y puede ser evaluada ${ }^{[8]}$ de acuerdo a la expresión (5) donde, $q$ representa la cantidad total de carga almacenada en la capa pasiva y $\imath$ el grado de transformación alcanzado al potencial de electrodo E.

$$
\begin{gathered}
\mathrm{Fe}_{3} \mathrm{O}_{4}+\mathrm{OH}^{-}+\mathrm{H}_{2} \mathrm{O} \longrightarrow 3 \mathrm{FeOOH}+\mathrm{e}^{-} \\
\mathrm{C}_{2}=\mathrm{q} \frac{\partial \theta}{\partial \mathrm{E}}
\end{gathered}
$$

El valor de $\mathrm{C}_{2}$ decrece para las disoluciones con LR y LG, disminución que es más significativa en presencia de aluminatos, lo cual indica que la capa pasiva tiene un espesor menor (menor valor de $q$ en la ecuación (5)) que en la disolución de referencia. Este resultado confirma lo anteriormente discutido a propósito de la corriente de pico de formación de la magnetita (figuras 2 a) y 3 a)), que es menor en presencia de lodos o aluminatos.

\section{CONCLUSIONES}

Los resultados expuestos anteriormente permiten concluir lo siguiente:
- Pequeñas adiciones de LG y LR son capaces de inhibir la corrosión en medio alcalino clorurado porque, previsiblemente, inducen la formación de una película adsorbida que bloquea la superficie del electrodo metálico.

- $\mathrm{El} \mathrm{Al}^{3+}$ es capaz de inhibir la corrosión de modo similar a LR y LG. Estos resultados sugieren que el efecto inhibidor es debido a la adsorción de los aluminatos sobre el electrodo creando una capa protectora tipo gel que no permanece al extraer el electrodo de la disolución y lavarlo.

- La impedancia global del sistema es mayor en presencia de $\mathrm{Al}^{3+}$ y disminuye a medida que decrece el contenido de $\mathrm{Al}^{3+}$ en la disolución (con LR y LG), lo que revela la importancia del papel que juegan los aluminatos en el efecto inhibidor ejercido por los lodos.

En general, los resultados obtenidos muestran cambios en el comportamiento electroquímico de la película pasiva formada en presencia de $\mathrm{Al}^{3+}$, cambios asociados a un cierto poder inhibidor por formación de una capa pasiva altamente resistiva y reducción de la corriente crítica de pasivación.

\section{Agradecimientos}

Los autores desean agradecer al Ministerio de Ciencia y Tecnología por la financiación concedida a través del proyecto BIA 2007-66491-C2-1.

\section{REFERENCIAS}

[1] B. Díaz, S. Joiret, M. Keddam, X.R. Nóvoa, M.C. Pérez y H. Takenouti, Electrochim. Acta 49 (2004) 3.039.

[2] C.M. Abreu, M.J. Cristóbal, L.Freire, X.R. Nóvoa, G. Pena y M.C. Pérez, J. Br. Chem. Soc. 6 (2007) 1.158. 
[3] E. López, B. Soto, M.Arias, A. Nuñez, A.D. Rubinos y M.T. Barral, Water Res. 32 (1998) 1314.

[4] J.P. Gonçalves, W.A. Moura y D.C. Coitinho dal Molin, Ambiente Construido 2,1 (2002) 53.

[5] J.M.S. Moreira, M.N. Freire y J.N.F. Holanda, Cerámica 49 (2003) 262.

[6] A. Collazo, M.J. Cristóbal, X.R. Nóvoa, G. Pena y M.C. Perez, J. ASTM Int. 3, 2 (2006) 1.

[7] M. Cabeza, A. Collazo, X.R. Nóvoa y M.C. Pérez, J. Corros. Sci. Eng. 6 (2003) CO77

[8] C. Andrade, P. Merino, X. R. Nóvoa, M. C. Pérez y L. Soler, Mater. Sci. Forum 192-194 (1995) 891.

[9] M. Pourbaix, Atlas d'équilibres électrochimiques, Gauthier-Villars Ed. Paris, (1963) p. 170.
[10] P. Pedeferri, Construc. Build. Mater. 10 (1996) 391.

[11] D. Izquierdo, C. Alonso, C. Andrade y M. Castellote, Electrochim. Acta 49 (2004) 2.731.

[12] S. Joiret, M.Keddam, X.R. Nóvoa, M.C. Pérez, C.Rangel y H. Takenouti, Cem. Concr. Compos. 24 (2002) 7.

[13] C.Andrade, M. Keddam, X.R. Nóvoa, C.M. Rangel y H.Takenouti, Electrochim. Acta 46 (2001) 3.905.

[14] C.M. Abreu, M.J. Cristobal, R. Losada, X.R. Nóvoa, G. Pena y M.C. Pérez, J. Electroanal. Chem. 572 (2004) 335.

[15] C. Exartier, S. Maximovitch y B. Baroux, Corros. Sci. 46 (2004) 1.777. 